\title{
Analysis of the humoral immune responses among cynomolgus macaque naturally infected with Reston virus during the 1996 outbreak in the Philippines
}

Satoshi Taniguchi ${ }^{1,2}$, Yusuke Sayama ${ }^{1,3}$, Noriyo Nagata ${ }^{4}$, Tetsuro Ikegami ${ }^{5}$, Mary E Miranda ${ }^{6}$, Shumpei Watanabe ${ }^{1}$ Itoe lizuka ${ }^{1,7}$, Shuetsu Fukushi ${ }^{1}$, Tetsuya Mizutani ${ }^{1}$, Yoshiyuki Ishii ${ }^{2}$, Masayuki Saijo ${ }^{1}$, Hiroomi Akashi ${ }^{8}$, Yasuhiro Yoshikawa ${ }^{2}$, Shigeru Kyuwa ${ }^{2}$ and Shigeru Morikawa ${ }^{*}$

\begin{abstract}
Background: Ebolaviruses induce lethal viral hemorrhagic fevers (VHFs) in humans and non-human primates, with the exceptions of Reston virus (RESTV), which is not pathogenic for humans. In human VHF cases, extensive analyses of the humoral immune responses in survivors and non-survivors have shown that the IgG responses to nucleoprotein (NP) and other viral proteins are associated with asymptomatic and survival outcomes, and that the neutralizing antibody responses targeting ebolaviruses glycoprotein $\left(\mathrm{GP}_{1,2}\right)$ are the major indicator of protective immunity. On the other hand, the immune responses in non-human primates, especially naturally infected ones, have not yet been elucidated in detail, and the significance of the antibody responses against NP and $\mathrm{GP}_{1,2}$ in RESTV-infected cynomolgus macaques is still unclear. In this study, we analyzed the humoral immune responses of cynomolgus macaque by using serum specimens obtained from the RESTV epizootic in 1996 in the Philippines to expand our knowledge on the immune responses in naturally RESTV-infected non-human primates.

Results: The antibody responses were analyzed using IgG-ELISA, an indirect immunofluorescent antibody assay (IFA), and a pseudotyped VSV-based neutralizing (NT) assay. Antigen-capture (Ag)-ELISA was also performed to detect viral antigens in the serum specimens. We found that the anti-GP 1,2 responses, but not the anti-NP responses, closely were correlated with the neutralization responses, as well as the clearance of viremia in the sera of the RESTV-infected cynomolgus macaques. Additionally, by analyzing the cytokine/chemokine concentrations of these serum specimens, we found high concentrations of proinflammatory cytokines/chemokines, such as IFNY, IL8, $\mathrm{IL}-12$, and MIP1a, in the convalescent phase sera.
\end{abstract}

Conclusions: These results imply that both the antibody response to $\mathrm{GP}_{1,2}$ and the proinflammatory innate responses play significant roles in the recovery from RESTV infection in cynomolgus macaques.

Keywords: Ebola, Ebolavirus, Reston virus, Reston ebolavirus, Filovirus, Zoonosis, Humoral immune response, Cynomolgus macaque, Cytokine, Antibody

\footnotetext{
* Correspondence: morikawa@nih.go.jp

'Special Pathogens Laboratory, Department of Virology 1, National Institute of Infectious Diseases, 4-7-1 Gakuen, Musashimurayama, Tokyo 208-0011, Japan

Full list of author information is available at the end of the article
} 


\section{Background}

The family Filoviridae includes three genera, Ebolavirus, Marburgvirus, and Cuevavirus. The genus Ebolavirus currently has five members: Bundibugyo virus (BDBV), Ebola virus (EBOV), Reston virus (RESTV), Sudan virus (SUDV), and Taï Forest virus [1]. Filoviruses induce lethal viral hemorrhagic fevers (VHFs) in both humans and nonhuman primates, while RESTV infection in humans is probably subclinical, yet it also causes highly lethal VHF in macaques [2,3]. RESTV epizootics among cynomolgus macaques emerged in 1989, 1990, 1992, and 1996. In all of these epizootics, the cynomolgus macaques originated in a single primate breeding facility in the Philippines [4]. Although the natural reservoir of RESTV remains unknown, RESTV was isolated from pigs in the Philippines, in addition to porcine reproductive and respiratory syndrome virus (PRRSV) and porcine circovirus type-2 in 2008 [5]. Considering the social impact of ebolaviruses, it is important to understand the endemic and epizootic status of RESTV in the Philippines.

In this study, we investigated the antibody responses of cynomolgus macaques that could be dead-end hosts for RESTV. Using serum specimens collected from cynomolgus macaques during a RESTV outbreak in the Philippines in 1996, we attempted to elucidate the significance of neutralizing antibodies to RESTV in viral clearance. We have previously established an enzymelinked immunosorbent assay (ELISA) and an indirect immunofluorescent antibody assay (IFA) specific for RESTV nucleoprotein (NP) [6-8]. These assays are useful tools for investigating the signs of RESTV infection in cynomolgus macaques. In human cases, antibody responses against ebolaviruses have been analyzed extensively: IgG responses to NP and other structural proteins (e.g., VP40 and VP35) have been shown to correlate with asymptomatic and surviving cases, and neutralizing antibody responses targeting the ebolaviruses glycoprotein $\left(\mathrm{GP}_{1,2}\right)$ appear to be the major indicator of protective immunity [9].

On the other hand, proinflammatory cytokines/chemokines are known to play a major role in the pathogenesis of ebolaviruses infections in various species. Previous studies have shown an uncontrolled secretion of proinflammatory cytokines/chemokines to contribute to a fatal outcome in EBOV-infected humans [10] and cynomolgus macaques [11]. Strong proinflammatory cytokine/chemokine responses are also observed in convalescent or asymptomatic cases [12,13]. In RESTVinfected cynomolgus macaques, high viremia has been shown to induce the secretion of proinflammatory cytokines/chemokines [14]. However, there have so far only been a limited number of studies on the impact of proinflammatory cytokine/chemokine responses in the convalescent phase of RESTV infection.
In this study, we grouped the cynomolgus macaque samples based on the presence of RESTV NP-antigen in sera and analyzed the antibody reactions and cytokine/ chemokine inductions to evaluate the presence of neutralizing antibody to RESTV. We found that the anti$\mathrm{GP}_{1,2}$ responses, but not the anti-NP responses, were closely correlated with the neutralization antibody responses, as well as the clearance of viremia, in the sera of RESTV-infected cynomolgus macaques. Additionally, a high concentration of proinflammatory cytokines/ chemokines was detected in the convalescent phase specimens. These data suggest that both the anti-GP ${ }_{1,2}$ responses and proinflammatory cytokines/chemokines play significant roles in the recovery from RESTV infection in cynomolgus macaques.

\section{Results}

RESTV NP-and $\mathrm{GP}_{1,2}$-specific antibodies, neutralizing antibody responses, and the viral antigens in the cynomolgus macaque sera from the 1996 RESTV epizootic Twenty-seven serum samples derived from cynomolgus macaques that were either found already dead or had been euthanized at the facility were available. The presence of RESTV NP antigens was evaluated by antigen-capture ELISA [15] or immunohistochemistry [3], while that of anti-RESTV NP IgG was evaluated using IgG ELISA and IFA methods [6-8]. RESTV NP antigens were detected in the liver in \# 2182, 2612, 2615, 2669, 2739, 2921, 2644 and 2728, while RESTV NP was detected by antigen capture ELISA in the sera of \#2182, 2612, 2408, 2615, 2669, 2739, 2921, 2721 and 2972. We therefore assumed that these cynomolgus macaques had suffered from the acute viremic phase of the disease. Seventeen of the 27 samples (\#2408, 2615, 2669, 2739, 2921, 2728, 2180, 2181, 2189, 2190, 2191, 2195, 2404, 2693, 2696, 2713 and 2194) were positive for anti-NP IgG in IgG ELISA, while these samples all reacted in IFA. On the other hand, two samples (\#2644 and 2719) were only positive in IFA. We considered the samples as anti-NP IgG-positive when either ELISA or IFA showed positive reaction. As a result, a total of 19 samples had anti-NP IgG. Cynomolgus macaques with anti-NP IgG consisted of NP antigen-positive (\#2408, 2615, 2669, 2739, 2921, 2644 and 2728) and NP antigennegative groups (\#2180, 2181, 2189, 2190, 2191, 2195, 2404, 2693, 2696 and 2713).

In order to examine whether the sera contained anti$\mathrm{GP}_{1,2}$ antibodies, we employed a $\mathrm{GP}_{1,2}$-specific ELISA and IFA [16]. RESTV $\mathrm{GP}_{1,2} \Delta \mathrm{TM}$ prepared by a baculovirus expression system and RESTV $\mathrm{GP}_{1,2}$-expressing $\mathrm{HeLa}$ cells were used as antigens for $\mathrm{GP}_{1,2}$-specific ELISA and IFA, respectively. Anti-RESTV $\mathrm{GP}_{1,2}$ IgG were detected in 10 (\#2180, 2181, 2189, 2190, 2191, 2195, 2404, 2693, 2696 and 2713) out of the 27 serum samples according to ELISA (37\%), whereas the 
remaining 17 samples (63\%) showed negative reactions. Nine serum samples positive for $\mathrm{GP}_{1,2}$ antibodies in the IgG-ELISA also showed positive reactions in the IFA, while one serum sample (\#2194) was only positive in the IFA. Serum samples showing positive reactions in either the $\mathrm{GP}_{1,2}$-specific IgG-ELISA or IFA were considered to be anti- $\mathrm{GP}_{1,2}$ positive $(11 / 27,41 \%$, Table 1$)$. Interestingly, the sera derived from cynomolgus macaques in the acute viremic phase did not contain any detectable anti-GP ${ }_{1,2}$ IgG, although they often contained anti-NP IgG.

We next attempted to detect the neutralization (NT) antibody response in the sera of RESTV-infected cynomolgus macaques. The VSV pseudotype RESTV $\mathrm{GP}_{1,2}$ (VSV-RESTV-GP $1,2 /$ GFP) was used for the NT assay [17]. Twelve serum samples $(12 / 27,44 \%)$ neutralized the
VSV-RESTV-GP ${ }_{1,2} /$ GFP infection, with NT titers ranging from 80 and 640 (\#2721, 2972, 2180, 2181, 2189, 2190, 2191, 2195, 2404, 2693, 2696 and 2713) (Table 1). The anti-GP 1,2 IgG were not detectable in \#2721 and \#2972 by IgG ELISA, while those samples both had a neutralizing activity. These two specimens showed a positive response for viral antigen in the Ag-capture ELISA and were thus considered to be collected in an early seroconversion phase.

All cynomolgus macaques at the facility were euthanatized regardless of clinical status and there was a possibility that some of the cynomolgus macaques had combined infection with simian hemorrhagic fever virus (SHFV) in the animal facility [18]. Therefore, immune responses against RESTV did not always reflect the

Table 1 Antibody responses and viremic status of Reston virus-infected cynomolgus macaques

\begin{tabular}{|c|c|c|c|c|c|c|c|c|c|}
\hline \multirow[t]{2}{*}{ Case ID } & \multicolumn{2}{|c|}{ Anti-NP IgG } & \multicolumn{2}{|c|}{ Anti-GP $P_{1,2} \lg G$} & \multirow[t]{2}{*}{ NT } & \multicolumn{2}{|c|}{ Ag-ELISA } & \multirow{2}{*}{$\begin{array}{l}\text { Overall } \\
\text { status }\end{array}$} & \multirow{2}{*}{$\begin{array}{l}\text { Dead or } \\
\text { **euthanizec }\end{array}$} \\
\hline & ELISA & IFA & ELISA & IFA & & liver & $\overline{\text { serum }}$ & & \\
\hline 2182 & - & $<80$ & - & $<80$ & - & + & + & $\mathrm{Ag}+\mathrm{NT}-$ & euthanized \\
\hline 2612 & - & $<80$ & - & $<80$ & - & + & + & $\mathrm{Ag}+\mathrm{NT}-$ & euthanized \\
\hline 2408 & + & 10240 & - & $<80$ & - & ${ }^{*} N D$ & + & $\mathrm{Ag}+\mathrm{NT}-$ & ${ }^{\#} \mathrm{NR}$ \\
\hline 2615 & + & 2560 & - & $<80$ & - & + & + & $\mathrm{Ag}+\mathrm{NT}-$ & euthanized \\
\hline 2669 & + & 2560 & - & $<80$ & - & + & + & $\mathrm{Ag}+\mathrm{NT}-$ & euthanized \\
\hline 2739 & + & 1280 & - & $<80$ & - & + & + & $\mathrm{Ag}+\mathrm{NT}-$ & euthanized \\
\hline 2921 & + & 1280 & - & $<80$ & - & + & + & $\mathrm{Ag}+\mathrm{NT}-$ & euthanized \\
\hline 2644 & - & 80 & - & $<80$ & - & + & ND & $\mathrm{Ag}+\mathrm{NT}-$ & euthanized \\
\hline 2728 & + & 1280 & - & $<80$ & - & + & ND & $\mathrm{Ag}+\mathrm{NT}-$ & euthanized \\
\hline 2721 & - & $<80$ & - & $<80$ & 80 & + & + & $\mathrm{Ag}+\mathrm{NT}+$ & euthanized \\
\hline 2972 & - & $<80$ & - & $<80$ & 160 & + & + & $\mathrm{Ag}+\mathrm{NT}+$ & dead \\
\hline 2180 & + & 1280 & + & 320 & 80 & - & - & $\mathrm{Ag}-\mathrm{NT}+$ & dead \\
\hline 2181 & + & 80 & + & 80 & 320 & - & - & $\mathrm{Ag}-\mathrm{NT}+$ & dead \\
\hline 2189 & + & 1280 & + & 320 & 320 & - & - & $\mathrm{Ag}-\mathrm{NT}+$ & NR \\
\hline 2190 & + & 160 & + & 640 & 640 & - & - & $\mathrm{Ag}-\mathrm{NT}+$ & NR \\
\hline 2191 & + & 640 & + & 320 & 320 & - & - & $\mathrm{Ag}-\mathrm{NT}+$ & NR \\
\hline 2195 & + & 2560 & + & 640 & 320 & - & - & $\mathrm{Ag}-\mathrm{NT}+$ & NR \\
\hline 2404 & + & 1280 & + & 160 & 160 & - & - & $\mathrm{Ag}-\mathrm{NT}+$ & dead \\
\hline 2693 & + & 160 & + & $<80$ & 320 & - & - & $\mathrm{Ag}-\mathrm{NT}+$ & euthanized \\
\hline 2696 & + & 2560 & + & 160 & 320 & - & - & $\mathrm{Ag}-\mathrm{NT}+$ & euthanized \\
\hline 2713 & + & 5120 & + & 320 & 160 & - & - & $\mathrm{Ag}-\mathrm{NT}+$ & euthanized \\
\hline 2719 & - & 80 & - & $<80$ & - & & & ND & NR \\
\hline 832 & - & $<80$ & - & $<80$ & - & & & ND & NR \\
\hline 888 & - & $<80$ & - & $<80$ & - & & & ND & NR \\
\hline 1134 & - & $<80$ & - & $<80$ & - & & & ND & NR \\
\hline 2636 & - & $<80$ & - & $<80$ & - & & & $\mathrm{ND}$ & euthanized \\
\hline 2194 & + & 5120 & - & 80 & - & & & $\mathrm{ND}$ & dead \\
\hline \multirow[t]{2}{*}{ No. of positive samples } & $17 / 27$ & $19 / 27$ & $10 / 27$ & $10 / 27$ & $12 / 27(44 \%)$ & $11 /$ & $52 \%)$ & & \\
\hline & \multicolumn{2}{|c|}{$19 / 27(70 \%)$} & \multicolumn{2}{|c|}{$11 / 27(41 \%)$} & & & & & \\
\hline
\end{tabular}

${ }^{*} N D$ : not determined, ${ }^{* *}$ euthanized: monkeys were euthanized regardless of clinical manifestation. ${ }^{*} N R$ : not recorded, $A g+:$ antigen positive, $A g$-: antigen negative, $N T+$ : neutralization antibody positive, $N T$-: neutralization antibody negative. The specimens with case IDs written in italics were considered in acute phase. The specimens with case IDs written in bold were considered in convalescent phase. 
clinical manifestation. For these reasons, we defined "convalescent" or "non-convalescent" based only upon serological findings.

It is noteworthy that, among the serum samples that were positive for viral antigen and negative for the NT antibody $(\mathrm{Ag}+, \mathrm{NT}-)$, all nine serum samples were negative for anti-GP $\mathrm{G}_{1,2}$ antibodies, whereas only two samples were negative for anti-NP antibodies. On the other hand, all of the ten serum samples that were negative for viral antigen and positive for the NT antibody (Ag -, NT +) were positive for both anti-GP $\mathrm{P}_{1,2}$ and anti-NP antibodies. This finding indicates that the anti- $\mathrm{GP}_{1,2}$ antibody may therefore increase in cynomolgus macaques in the convalescent phase, while anti-GP $\mathrm{P}_{1,2}$ antibody is rarely detectable in the acute viremic phase of infection.

\section{Multiplex assay for cytokines and chemokines in the cynomolgus macaque sera}

Ebola virus infection triggers the expression of several proinflammatory cytokines/chemokines $[11,19,20]$. To examine whether the convalescence from the RESTV infection correlates with the circulating proinflammatory cytokines/chemokines, eleven RESTV-infected cynomolgus macaque serum samples were subjected to a multiplex cytokine analysis. Since we do not know when the infection occurred for each cynomolgus macaque, it is still unclear whether the sera represented an early or late stage of infection. We used seven convalescent phase sera (Ag-, NT +: \#2404, 2181, 2189, 2693, 2696, 2713, 2180), and four acute viremic phase sera ( $\mathrm{Ag}+$, NT -: \#2182, 2612, 2739, 2921). Among the 27 serum samples, only these 11 serum samples were available for multiplex assay. Since the sera were heat-inactivated at $56^{\circ} \mathrm{C}$ for 30 min prior to being subjected to the multiplex analysis, some cytokines, such as GM-CSF and IL-2, which were previously shown to be elevated in some RESTV infected cynomolgus macaques [14], could not be measured in the assay.

We found that concentrations of several proinflammatory cytokines/chemokines (e.g., IFN $\gamma$, IL8, IL-12, IL-1ra, and MIP $1 \alpha$ ) were significantly higher in convalescent than in acute phase sera (Figure 1). This observation is similar to the previous studies showing elevated concentrations of proinflammatory cytokines/chemokines in the convalescent or asymptomatic human cases $[12,13]$. In contrast, the concentrations of the five cytokines/chemokines (e.g., IFN $\alpha$, IP-10, MIP1 $\beta$, IL-6, and TNF $\alpha$ ) did not differ significantly between the two groups (Figure 2 ). Furthermore, the concentration of MCP-1, one of the proinflammatory chemokines, was lower in the convalescent than in the non-convalescent sera (Figure 2). These data indicated that IFN- $\gamma$, IL-8, IL-12, IL-1ra or MIP1 $\alpha$ might therefore be involved with the host immune responses in the convalescent phase of RESTV infection.

\section{Discussion}

We previously developed a RESTV NP-specific IgGELISA and IFA that proved to be useful for the seroepidemiological studies of cynomolgus macaques during the RESTV epizootic in the Philippines in 1996 [6-8]. The assays based on recombinant NP are sensitive for the detection of RESTV-specific antibodies. On the other hand, anti-GP ${ }_{1,2}$ antibodies are elicited in EBOVinfected human cases and are believed to have protective roles against lethal EBOV infection [21,22]. In the present study, in order to gain insight into the IgG responses during the recovery from infection with RESTV, anti-NP, anti-GP ${ }_{1,2}$, and neutralizing antibodies and the level of viremia in the serum specimens were analyzed. The data presented herein showed that the anti- $\mathrm{GP}_{1,2}$ response, rather than the anti-NP response, was correlated with both the lack in viremia and the neutralizing activities in the sera of RESTV-infected cynomolgus macaques. There may be at least two possibilities for the lack of anti-GP $\mathrm{P}_{1,2}$ IgG in the acute phase samples. It is known that soluble GP (sGP), which does not contain membrane anchor, is secreted during ebolaviruses infection, and it can absorb the anti- $\mathrm{GP}_{1,2}$ antibodies [23]. The other possibility is that apoptosis of lymphocytes is induced during RESTV infection and the resulting host immune responses may thus be abrogated. Although the precise mechanism of action is still unknown, it is likely that no IgG responses to RESTV $\mathrm{GP}_{1,2}$ are induced in the cynomolgus macaques during the acute phase of infection.

Since the cynomolgus macaques at the facility where the RESTV epizootic occurred were euthanized, sequential serum specimens from each cynomolgus macaque were not available. It is also difficult to determine when each cynomolgus macaque became infected with RESTV. We found that three specimens that have antiGP antibodies were obtained from dead monkeys (\#2180, 2181 and 2194), however, it is difficult to conclude whether RESTV infection caused their death because of the possibility of succumbing to infection by SHFV or some other agents. It is predicted that, among the serum samples examined here, nine were acute phase samples because they were positive in Ag-ELISA or immunohistochemistry [3,15] (Table 1). On the other hand, all but one (serum \#2194) of the anti-GP ${ }_{1,2^{-}}$ positive serum samples were $\mathrm{Ag}$ negative. Therefore, these cynomolgus macaques were considered to be in the convalescent phase of RESTV infection. In this regard, the presence of the anti- $\mathrm{GP}_{1,2}$ antibody is thought to be a useful indicator for convalescence in cynomolgus macaques infected with RESTV.

Aberrant proinflammatory cytokines/chemokines are a significant factor implicated in the disease progression of EBOV- and SUDV-infected human cases and experimentally 

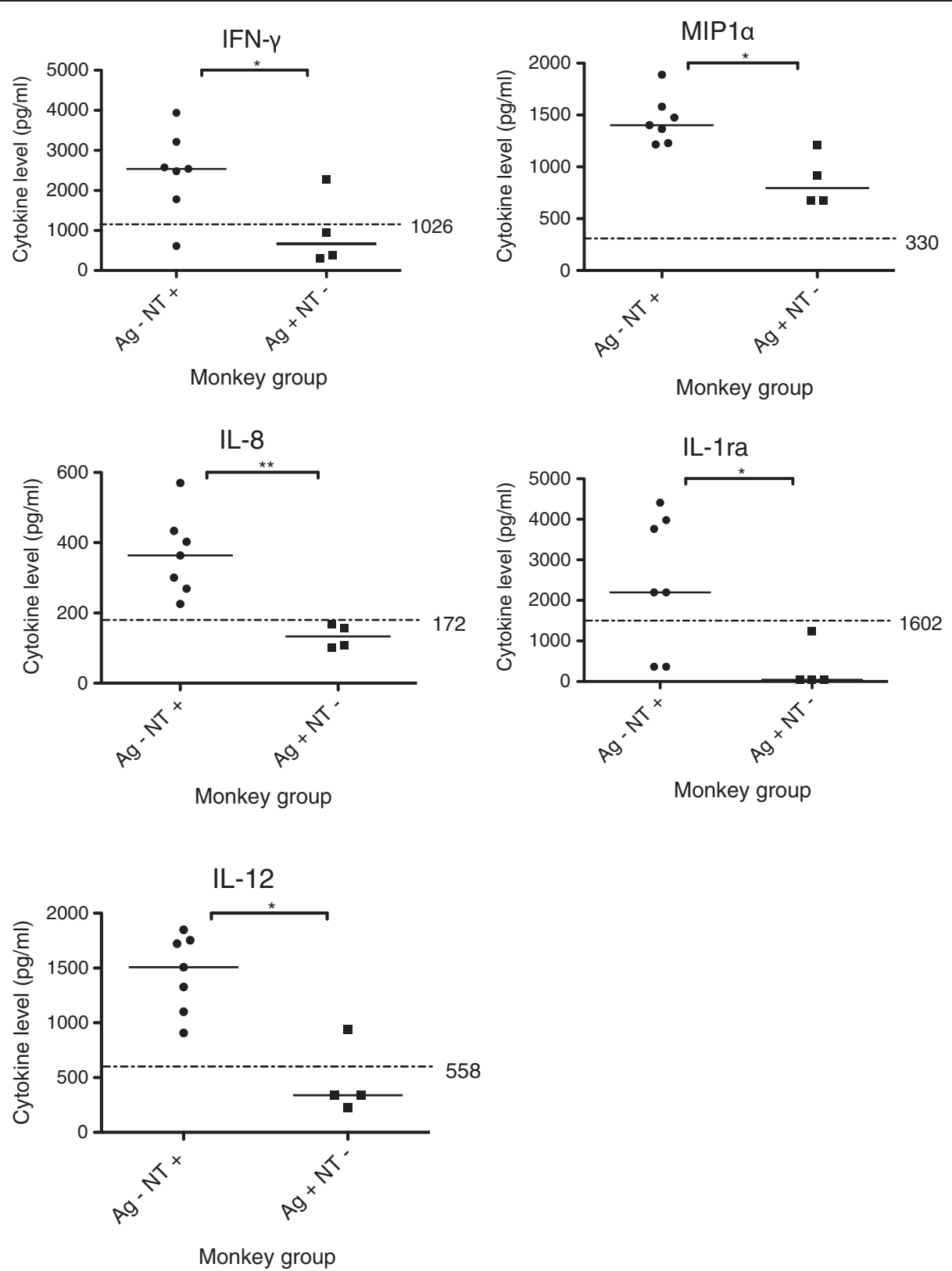

Figure 1 Profiles of the serum cytokine and chemokine concentrations in Reston virus-infected cynomolgus macaques. The serum concentrations of IFN- $\gamma$, IL-8, IL-12, MIP1a, and IL-1 ra. The concentrations of these proinflammatory cytokines/chemokines (IFN- $\gamma$, IL-8, IL-12, MIP1 a) and the anti-inflammatory cytokine (IL-1 ra) were significantly higher in convalescent (Ag - NT +) than in non-convalescent sera (Ag + NT -). Each dot represents one sample, and dashes (-) represent the median values. ${ }^{*}$ indicates $p<0.05$, ** indicates $p<0.005$ (Mann Whitney test). Broken lines and the numbers written aside indicate the average concentrations of cytokine and chemokine in negative control cynomolgus macaques $(n=13)$.

infected cynomolgus macaques [10,11,24]. In addition, a balanced proinflammatory response is believed to be a critical factor for determining the disease outcome $[25,26]$. We focused on the circulating inflammatory cytokines/chemokines in RESTV-infected cynomolgus macaques and examined their relationship with convalescence. We thus found the concentrations of several proinflammatory cytokines/ chemokines, such as IFNy, IL8, IL-12, and MIP1 $\alpha$, to be significantly higher in convalescent sera than in nonconvalescent sera. Gupta et al. (2012) recently demonstrated that convalescent serum samples obtained from BDBVinfected human cases include high concentrations of IL- $1 \alpha$, IL1 $\beta$, IL6, TNF $\alpha$, and MCP-1 [13]. Although the exact profiles of proinflammatory cytokines/chemokines shown in our study are different from those reported by Gupta et al., these differences are considered most likely to be due to 

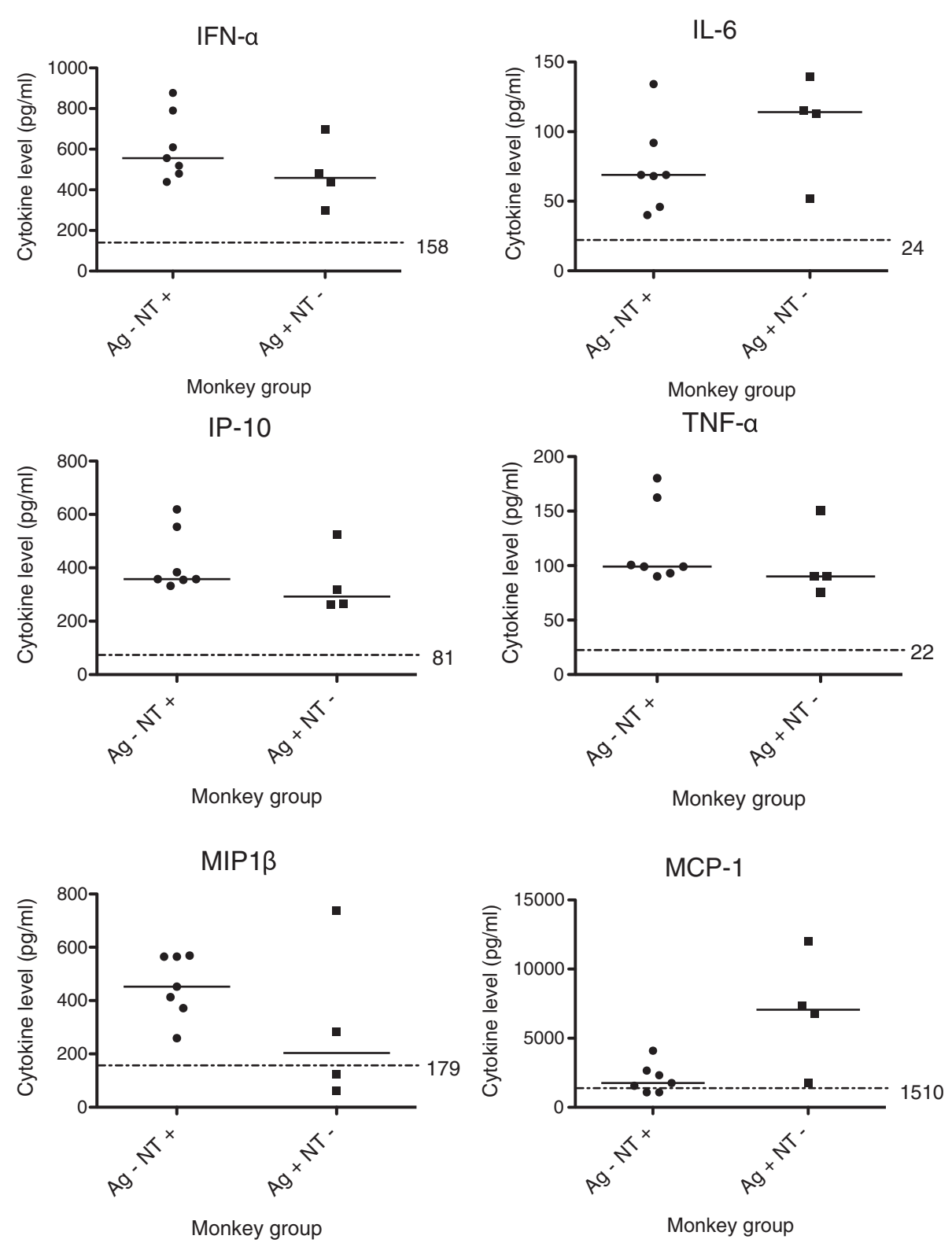

Figure 2 Profiles of the serum cytokine and chemokine concentrations in Reston virus-infected cynomolgus macaques. The IFN- $a$, IP-10, TNF-a, MIP1 $\beta$, IL-6 and MCP-1 concentrations. The serum concentrations of these cytokines/chemokines (IFN-a, IP-10, TNF-a, MIP1 $\beta$ ) did not differ significantly between the convalescent cases (Ag - NT +) and the non-convalescent cases (Ag + NT -). The concentration of the anti-inflammatory cytokine, MCP-1, was lower in convalescent than in non-convalescent cynomolgus macaques (Mann Whitney test). Each dot represents one sample, and dashes (-) represent the median values. Broken lines and the numbers written aside indicate the average concentrations of cytokine and chemokine in negative control cynomolgus macaques $(n=13)$.

differences among ebolaviruses (RESTV vs. BDBV), host species (cynomolgus macaques vs. humans), and differences related to the disease phase when the samples were obtained. It is possible that the upregulation of the proinflammatory innate immune responses contributed to the recovery from RESTV infection in cynomolgus macaques.

In rhesus monkeys experimentally infected with a lethal dose of EBOV, anti-inflammatory cytokines, such as IL-13 and IL-1ra, are highly elevated in the acute phase [11]. In human Ebola VHF patients, increased concentrations of IL-10 and IL-1ra have been shown in fatal cases, thus suggesting that the mixed anti-inflammatory response syndrome (MARS) contributes to the pathogenesis of the hemorrhagic fever caused by ebolaviruses. Since all of the cynomolgus macaques involved in the epizootic were euthanized at the affected facility, the actual fate of the cynomolgus macaques was not clear, and some might have survived the infection. Our data obtained using the sera 
from cynomolgus macaques in the RESTV epizootic showed higher IL-1ra responses in the convalescent phase than in the non-convalescent phase (Figure 1). There were no significant differences in the concentration of IL-10 between the two groups (data not shown). This suggests that, unlike other ebolaviruses infections, RESTV does not induce MARS, which is characterized by an elevated induction of IL1ra in the acute phase.

In conclusion, we have shown that the anti-GP ${ }_{1,2}$ responses, rather than the anti-NP responses, in cynomolgus macaques naturally infected with RESTV were specifically detected in the convalescent stage of RESTV infection. In addition, a high concentration of proinflammatory cytokines/chemokines was observed in the convalescent phase. Therefore, the anti-GP $\mathrm{G}_{1,2}$ response and the upregulation of the specific proinflammatory response might be useful indicators of convalescence from RESTV infection in cynomolgus macaques.

\section{Conclusions}

In this study, we analyzed the humoral responses in cynomolgus macaque serum samples collected during the 1996 Reston outbreak in the Philippines and demonstrated that the anti-RESTV $\mathrm{GP}_{1,2}$ response and the proinflammatory innate response play significant roles in the convalescence from RESTV infection in cynomolgus macaques.

\section{Methods}

\section{Sera}

Twenty-seven cynomolgus macaque serum samples were obtained from the cynomolgus macaque facility in the Philippines where the 1996 RESTV epizootic occurred [27]. The serum specimens in the affected facility were collected under quarantine of the Philippines. Nineteen of the 27 samples were previously subjected to an antigen capture ELISA. Nine of the samples were found to be RESTV antigen-positive, and the remaining 10 were considered to be antigen-negative [15]. The serum specimens were treated at $56^{\circ} \mathrm{C}$ for 30 minutes and virus in the cynomolgus macaque serum samples were inactivated. As negative controls for the IgG-ELISA and IFA, we used serum samples from 102 cynomolgus macaques collected at the Tsukuba Primate Research Center (TPRC) in Japan. As positive controls for the IgG-ELISA and IFA, two rabbits were immunized four times with the histidine-tagged ectodomain of the RESTV glycoprotein $\left(\mathrm{GP}_{1,2}\right)$ (RESTV $\left.\mathrm{GP}_{1,2} \Delta \mathrm{TM}\right)$. The histidine-tagged RESTV GP ${ }_{1,2} \Delta$ TM of a 1996 RESTV [28] was prepared and purified as described below. The sera were collected from the rabbits, inactivated, and stored at $4^{\circ} \mathrm{C}$ until use. The experiments with animals were performed in accordance with the Animal Experimentation Guidelines of the National Institute of Infectious Diseases. The protocol was approved by the Institutional Animal Care and Use Committee of the institute (Permit number: 990163 and 109075).

\section{Expression and purification of the recombinant RESTV $\mathrm{GP}_{1,2}$ ectodomain}

A recombinant baculovirus that expresses RESTV $\mathrm{GP}_{1,2} \Delta \mathrm{TM}$ was used to prepare recombinant RESTV $\mathrm{GP}_{1,2}$ for the IgG-ELISA [16]. Briefly, a recombinant baculovirus carrying the ectodomain of RESTV $\mathrm{GP}_{1,2}$ (DDBJ accession no. AB050936) with histidine-tag sequences at its 3'-terminus was infected into Tn5 cells at a multiplicity of infection (moi) of 1 . The cells were collected, washed with PBS, and then lysed in PBS containing $1 \%$ Nonidet P40 (NP-40) on ice for $15 \mathrm{~min}$. After being centrifuged, the recombinant RESTV $\mathrm{GP}_{1,2} \Delta \mathrm{TM}$ was purified with $\mathrm{Ni}^{2+}$-agarose beads (QIAGEN, Hilden, Germany) and His Bind Kits (Novagen, Darmstadt, Germany). The purified recombinant RESTV $\mathrm{GP}_{1,2} \Delta \mathrm{TM}$ was used for the IgG-ELISA specific for RESTV $\mathrm{GP}_{1,2}$. Lysates of Tn5 cells infected with baculovirus with a deleted polyhedrin gene, Ac- $\Delta \mathrm{P}$, were similarly processed and then used as negative control antigen in the IgG-ELISA described below.

\section{RESTV GP $_{1,2}$-specific IgG-ELISA}

Ninety-six well plates were coated with the RESTV $\mathrm{GP}_{1,2} \Delta \mathrm{TM}$ or with negative control antigen in $100 \mu \mathrm{l}$ of PBS and incubated overnight at $4^{\circ} \mathrm{C}$. The plates were washed three times with PBS containing 0.05\% Tween 20 (PBS-T), and then $200 \mu \mathrm{l}$ of PBS-T containing 5\% skim milk (SKIM-PBS-T) was added to each well and incubated for $2 \mathrm{hr}$ at $37^{\circ} \mathrm{C}$. The cynomolgus macaque sera were diluted at 1:100, 1:400, 1:1,600, and 1:6,400 in SKIM-PBS-T, and the hyperimmune rabbit sera were four-fold serially diluted from 1:1,000 to $1: 64,000$ in SKIM-PBS-T. One hundred microliters of each serum dilution was added to the antigen-coated wells and incubated for $1 \mathrm{hr}$ at $37^{\circ} \mathrm{C}$. After they were washed three times with PBS-T, the wells were further reacted with either HRP-conjugated goat anti-human $\operatorname{IgG}(\mathrm{H}+\mathrm{L})$ (Lot:60504974, ZyMED) or HRP-conjugated goat antirabbit IgG $(\mathrm{H}+\mathrm{L})$ (Lot:398581A, ZyMED) at a dilution of 1:1,000 in SKIM-PBS-T. After being washed three times again with PBS-T, the ABTS substrate (Roche Diagnostics) was added to the wells. Then, the plates were incubated for 30 minutes at $37^{\circ} \mathrm{C}$, and the OD values of the wells at $405 \mathrm{~nm}$ were measured. Adjusted OD values were calculated by subtracting the OD value of the wells coated with the negative control antigen from that of the wells coated with RESTV $\mathrm{GP}_{1,2} \Delta \mathrm{TM}$.

\section{RESTV NP-specific IgG-ELISA}

The NP-specific IgG-ELISA, which is similar to the $\mathrm{GP}_{1,2}$-specific ELISA except for the purified recombinant 
RESTV NP with a histidine tag at the C-terminus, has been previously reported $[6,16]$.

\section{ELISA index and determination of the cut-off value for the IgG-ELISA}

The sum of the OD values of serum dilutions at 1:100, $1: 400,1: 1,600$, and 1:6,400 for each specimen was calculated and designated as an "ELISA index" in the IgG-ELISA. The mean plus three standard deviations (SD) of the ELISA indices for the IgG-ELISAs was calculated using serum samples from uninfected TPRC cynomolgus macaques and was used as the cut-off value for the IgG-ELISAs.

\section{Indirect immunofluorescent antibody assay (IFA) specific for RESTV NP and GP ${ }_{1,2}$}

The IFA specific for RESTV NP was reported previously [8]. In the present study, a RESTV $\mathrm{GP}_{1,2}$-specific IFA was established using stably RESTV $\mathrm{GP}_{1,2}$-expressing HeLa cells. HeLa cell line was purchased from the American Type Culture Collection and used. The RESTV GP $_{1,2}$ cDNA of a 1996 RESTV was subcloned into a mammalian expression plasmid, pKS336, to gen-

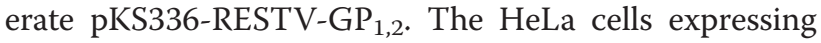
RESTV GP $_{1,2}$ were selected in a medium containing $2 \mu \mathrm{g} / \mathrm{ml}$ of blasticidin-S-hydrochloride (Sigma, St. Lois, MO) after transfection with pKS336-RESTV-GP ${ }_{1,2}$ using the FuGENE HD Transfection Reagent (Roche Diagnostics, Germany). The cells were trypsinized, washed with PBS, and mixed with normal HeLa cells, and were then spotted on 14 well Teflon-coated glass slides, air dried, and fixed with acetone at room temperature for $5 \mathrm{~min}$. The slides were stored at $-80^{\circ} \mathrm{C}$ until use.

The slides were thawed and dried just before use. The serum specimens were 2-fold serially diluted in PBS, and a $20 \mu \mathrm{l}$ aliquot of each dilution was applied to the wells of the antigen slides and incubated at $37^{\circ} \mathrm{C}$ for $1 \mathrm{hr}$ in a humidified chamber. Then the antigen slides were washed with PBS and reacted with $20 \mu \mathrm{l}$ per well of FITC-conjugated goat anti-human IgG $(\mathrm{H}+\mathrm{L})$ (ZyMax lot: 415460A, Invitrogen, CA, U.S.A.) for cynomolgus macaque sera and FITC-conjugated goat anti-rabbit IgG $(\mathrm{H}+\mathrm{L})$ (ZyMax lot: 402686A, Invitrogen, CA, U.S.A.) for rabbit hyperimmune sera at a dilution of 1:100. After incubation at $37^{\circ} \mathrm{C}$ for $1 \mathrm{hr}$, the slides were washed with PBS and covered with micro cover glasses. The slides were examined for the staining pattern under a fluorescent microscope. The antibody titer in the IFA was determined as the reciprocal of the highest dilution showing positive staining.

\section{RESTV neutralization (NT) assay using VSV-RESTV-GP 1,2 $^{/}$ GFP}

The VSV pseudotype bearing RESTV GP 1,2 , VSV-RESTV$\mathrm{GP}_{1,2} / \mathrm{GFP}$ was generated essentially according to the method described for the VSV pseudotype bearing SARSCoV S protein [29], except that pKS336-RESTV-GP 1,2 was used in the present study [17]. Briefly, $293 \mathrm{~T}$ cells were prepared in 24 well plates at $20-30 \%$ confluency. The cells were transfected with pKS336-RESTV-GP ${ }_{1,2}$ using FuGENE HD. The cells were then cultured for $24 \mathrm{hr}$ and inoculated with VSV $\Delta G^{*} / G F P$ pseudotyped with the VSV-G protein at a moi of 5, adsorbed for $1 \mathrm{hr}$ at $37^{\circ} \mathrm{C}$, and then washed with DMEM-5\% FCS and cultured for $24 \mathrm{hr}$. The culture supernatants were collected and centrifuged at 1,000 rpm to remove cell debris. Thereafter, the supernatants were stored at $-80^{\circ} \mathrm{C}$ as VSV-RESTV-GP ${ }_{1,2} /$ GFP. The infectivity titer of VSV-RESTV-GP 1,2 $_{2} /$ GFP, harboring the VSV $\Delta G^{*} / G F P$ genome, was determined by counting the number of GFP-positive cells under a fluorescent microscope upon infection into Vero E6 cells, as described previously. Briefly, VSV-RESTV-GP ${ }_{1,2} /$ GFP was $3.2\left(0.5 \log _{10}\right)$-fold serially diluted with DMEM-5\% FCS and then inoculated to Vero E6 cells seeded in 96 well culture plates. The cells were incubated at $37^{\circ} \mathrm{C}$ in a $\mathrm{CO}_{2}$ incubator for $24 \mathrm{hr}$. Then, GFP-positive cells were detected and counted under a fluorescent microscope (BZ-9000; KEYENCE, Osaka, Japan), and the infectious units (IU) of the pseudotyped VSV were calculated.

The serum samples were serially diluted in DMEM-5\% FCS, and a $50 \mu \mathrm{l}$ aliquot of each dilution was mixed with the same volume of DMEM-5\% FCS containing 1,000 IU of VSV-RESTV-GP $1,2 /$ GFP and incubated for $1 \mathrm{hr}$ at $37^{\circ} \mathrm{C}$. The mixture was inoculated into Vero E6 cells and incubated for $24 \mathrm{hr}$. The number of GFPpositive infected cells was counted, and serum dilutions with $50 \%$ neutralization $\left(\mathrm{NT}_{50}\right)$ were identified.

\section{Multiplex assay for cytokines and chemokines in the cynomolgus macaque sera}

Eleven RESTV-infected cynomolgus macaque serum samples were inactivated at $56^{\circ} \mathrm{C}$ for $30 \mathrm{~min}$, diluted 1:10 in the assay diluent supplied with the Human Cytokine 25-Plex antibody bead kit (Invitrogen, CA), and were subjected to a multiplex cytokine analysis using a Luminex 100 instrument (Luminex Co., Austin, TX) according to the manufacturer's instructions. This Human Cytokine 25-Plex antibody bead kit was previously used to cynomolgus macaque sera and the crossreactivity was confirmed [30]. As negative controls, we used sera from 13 cynomolgus macaques bred at the TPRC and investigated the cytokine concentrations of these serum samples.

\footnotetext{
Abbreviations

Ag: Antigen; BDBV: Bundibugyo ebolavirus; EBOV: Ebola virus; ELISA: Enzymelinked immunosorbent assay; $\mathrm{GP}_{1,2}$ : Glycoprotein; $\mathrm{GP}_{1,2} \Delta \mathrm{TM}$ : Ectodomain of the RESTV glycoprotein; VHF: Viral hemorrhagic fever;

IFA: Immunofluorescent antibody assay; MARS: Mixed anti-inflammatory response syndrome; NP: Nucleoprotein; NP-40: Nonidet P40;

NT: Neutralization; PBS-T: PBS containing 0.05\% Tween 20; RESTV: Reston
} 
virus; SD: Standard deviation; SHFV: Simian hemorrhagic fever virus; SKIMPBS-T: PBS-T containing 5\% skim milk; TPRC: Tsukuba Primate Research Center; VSV: Vesicular stomatitis Indiana virus; VSV-RESTV-GP $1,2 / G F P$ : VSV pseudotype bearing RESTV GP 1,2 .

\section{Competing interests}

The authors declare that they have no competing interests.

\section{Authors' contributions}

$\mathrm{ST}, \mathrm{TI}, \mathrm{SF}$, and SM designed the experiments and analyzed the experimental data. ST and SF prepared the manuscript. SM supervised the experiments and helped draft the manuscript. YS, SW, and II helped to perform the experiments. NN performed the multiplex assay. $Y Y$ and MM prepared the serum samples. TI, TM, YI, MS, HA and SK supervised the experiments. All authors have read and approved the final manuscript.

\section{Acknowledgements}

We are grateful to the staff at the Special Pathogens Laboratory, National Institute of Infectious Diseases, and at the Biomedical Science Laboratory, University of Tokyo.

This work was supported in part by a grant-in-aid from the Ministry of Health, Labor and Welfare of Japan (grants H22-shinkou-ippan-006) and the Japan Society for the Promotion of Science KAKENHI.

\section{Author details}

'Special Pathogens Laboratory, Department of Virology 1, National Institute of Infectious Diseases, 4-7-1 Gakuen, Musashimurayama, Tokyo 208-0011, Japan. ${ }^{2}$ Department of Biomedical Science, Graduate School of Agricultural and Life Sciences, University of Tokyo, 1-1-1 Yayoi, Bunkyo-ku, Tokyo 113-8657, Japan. ${ }^{3}$ Department of Virology, Tohoku University Graduate School of Medicine, 2-1 Seiryo-machi, Aoba-ku, Sendai, Japan. ${ }^{4}$ Department of Pathology, National Institute of Infectious Diseases, 4-7-1 Gakuen, Musashimurayama, Tokyo 208-0011, Japan. ${ }^{5}$ Department of Microbiology and Immunology, The University of Texas Medical Branch at Galveston, Mary Moody Northen Pavilion 3.206D, 301 University Boulevard Galveston, Texas, TX 77555-0436, USA. ${ }^{6}$ Veterinary Public Health Specialist, Aralia St., Ayala Westgrove Heights, Silang, Cavite 4118, Philippines. ${ }^{7}$ Faculty of Medicine, The University of Tokyo, Bunkyo-ku, Tokyo, Japan. ${ }^{8}$ Department of Veterinary Microbiology, Graduate School of Agricultural and Life Sciences, University of Tokyo, 1-1-1 Yayoi, Bunkyo-ku, Tokyo 113-8657, Japan.

Received: 15 June 2012 Accepted: 4 October 2012 Published: 11 October 2012

\section{References}

1. Kuhn JH, Becker S, Ebihara H, Geisbert TW, Jahrling PB, Kawaoka Y, Netesov SV, Nichol ST, Peters CJ, Volchkov VE, Ksiazek TG: Family_filoviridae. In Virus taxonomy - ninth report of the international committee on taxonomy of viruses. Edited by King Andrew MQ, Adams Michael J, Carstens Eric B, Lefkowitz Elliot J. London, United Kingdom: Elsevier/Academic Press; 2011:665-671.

2. Morikawa S, Saijo M, Kurane I: Current knowledge on lower virulence of Reston Ebola virus (in French: connaissances actuelles sur la moindre virulence du virus Ebola Reston). Comp Immunol Microbiol Infect Dis 2007, 30(5-6):391-398.

3. Ikegami T, Miranda ME, Calaor AB, Manalo DL, Miranda NJ, Niikura M, Saijo M, Une Y, Nomura Y, Kurane I, et al: Histopathology of natural Ebola virus subtype Reston infection in cynomolgus macaques during the Philippine outbreak in 1996. Exp Anim 2002, 51(5):447-455.

4. Miranda ME, Miranda NL: Reston ebolavirus in humans and animals in the Philippines: a review. J Infect Dis 2011, 204(Suppl 3):S757-S760.

5. Barrette RW, Metwally SA, Rowland JM, Xu L, Zaki SR, Nichol ST, Rollin PE, Towner JS, Shieh WJ, Batten B, et al: Discovery of swine as a host for the Reston ebolavirus. Science 2009, 325(5937):204-206.

6. Saijo M, Niikura M, Morikawa S, Ksiazek TG, Meyer RF, Peters CJ, Kurane I: Enzyme-linked immunosorbent assays for detection of antibodies to Ebola and Marburg viruses using recombinant nucleoproteins. J Clin Microbiol 2001, 39(1):1-7.

7. Ikegami T, Saijo M, Niikura M, Miranda ME, Calaor AB, Hernandez M, Manalo DL, Kurane I, Yoshikawa Y, Morikawa S: Immunoglobulin G enzyme-linked immunosorbent assay using truncated nucleoproteins of Reston Ebola virus. Epidemiol Infect 2003, 130(3):533-539.

8. Ikegami T, Saijo M, Niikura M, Miranda ME, Calaor AB, Hernandez M, Manalo DL, Kurane I, Yoshikawa Y, Morikawa S: Development of an immunofluorescence method for the detection of antibodies to ebola virus subtype Reston by the use of recombinant nucleoproteinexpressing HeLa cells. Microbiol Immunol 2002, 46(9):633-638.

9. Baize S, Leroy EM, Georges-Courbot MC, Capron M, Lansoud-Soukate Debre P, Fisher-Hoch SP, McCormick JB, Georges AJ: Defective humoral responses and extensive intravascular apoptosis are associated with fatal outcome in ebola virus-infected patients. Nat Med 1999, 5(4):423-426.

10. Wauquier N, Becquart P, Padilla C, Baize S, Leroy EM: Human fatal Zaire ebola virus infection is associated with an aberrant innate immunity and with massive lymphocyte apoptosis. PLoS Negl Trop Dis 2010, 4(10):e837.

11. Ebihara H, Rockx B, Marzi A, Feldmann F, Haddock E, Brining D, Lacasse RA, Gardner D, Feldmann H: Host response dynamics following lethal infection of Rhesus macaques with Zaire ebolavirus. J Infect Dis 2011, 204(Suppl 3):S991-S999.

12. Leroy EM, Baize S, Volchkov VE, Fisher-Hoch SP, Georges-Courbot MC, Lansoud-Soukate J, Capron M, Debre P, McCormick JB, Georges AJ: Human asymptomatic ebola infection and strong inflammatory response. Lancet 2000, 355(9222):2210-2215.

13. Gupta M, MacNeil A, Reed ZD, Rollin PE, Spiropoulou CF: Serology and cytokine profiles in patients infected with the newly discovered Bundibugyo ebolavirus. Virology 2012, 423(2):119-124.

14. Hutchinson KL, Villinger F, Miranda ME, Ksiazek TG, Peters CJ, Rollin PE: Multiplex analysis of cytokines in the blood of cynomolgus macaques naturally infected with ebola virus (Reston serotype). J Med Virol 2001, 65(3):561-566.

15. Ikegami T, Niikura M, Saijo M, Miranda ME, Calaor AB, Hernandez M, Acosta LP, Manalo DL, Kurane I, Yoshikawa Y, et al: Antigen capture enzymelinked immunosorbent assay for specific detection of Reston ebola virus nucleoprotein. Clin Diagn Lab Immunol 2003, 10(4):552-557.

16. Taniguchi S, Watanabe S, Masangkay JS, Omatsu T, Ikegami T, Alviola P, Ueda N, Iha K, Fujii H, Ishii Y, et al: Reston ebolavirus antibodies in bats, the Philippines. Emerg Infect Dis 2011, 17(8):1559-1560.

17. Sayama Y, Demetria C, Saito M, Azul RR, Taniguchi S, Fukushi S, Yoshikawa T, lizuka I, Mizutani T, Kurane I, et al: A seroepidemiologic study of Reston ebolavirus in swine in the Philippines. BMC Vet Res 2012, 8:82.

18. Rollin PE, Williams RJ, Bressler DS, Pearson S, Cottingham M, Pucak G, Sanchez A, Trappier SG, Peters RL, Greer PW, et al: Ebola (subtype Reston) virus among quarantined nonhuman primates recently imported from the Philippines to the United States. I Infect Dis 1999, 179(Suppl 1):S108-S114.

19. Hensley LE, Young HA, Jahrling PB, Geisbert TW: Proinflammatory response during ebola virus infection of primate models: possible involvement of the tumor necrosis factor receptor superfamily. Immunol Lett 2002, 80(3):169-179.

20. Baize S, Leroy EM, Georges AJ, Georges-Courbot MC, Capron M, Bedjabaga I, Lansoud-Soukate J, Mavoungou E: Inflammatory responses in ebola virus-infected patients. Clin Exp Immunol 2002, 128(1):163-168.

21. Wilson JA, Hevey M, Bakken R, Guest S, Bray M, Schmaljohn AL, Hart MK: Epitopes involved in antibody-mediated protection from ebola virus. Science 2000, 287(5458):1664-1666.

22. Prehaud C, Hellebrand E, Coudrier D, Volchkov VE, Volchkova VA, Feldmann $H$, Le Guenno B, Bouloy M: Recombinant ebola virus nucleoprotein and glycoprotein (Gabon 94 strain) provide new tools for the detection of human infections. J Gen Virol 1998, 79(Pt 11):2565-2572.

23. Volchkov VE, Volchkova VA, Slenczka W, Klenk HD, Feldmann H: Release of viral glycoproteins during ebola virus infection. Virology 1998, 245(1):110-119.

24. Sanchez A, Lukwiya M, Bausch D, Mahanty S, Sanchez AJ, Wagoner KD, Rollin PE: Analysis of human peripheral blood samples from fatal and nonfatal cases of ebola (Sudan) hemorrhagic fever: cellular responses, virus load, and nitric oxide levels. J Virol 2004, 78(19):10370-10377.

25. Bray M, Geisbert TW: Ebola virus: the role of macrophages and dendritic cells in the pathogenesis of ebola hemorrhagic fever. Int J Biochem Cell Biol 2005, 37(8):1560-1566.

26. Bray M, Mahanty S: Ebola hemorrhagic fever and septic shock. J Infect Dis 2003, 188(11):1613-1617

27. Miranda ME, Ksiazek TG, Retuya TJ, Khan AS, Sanchez A, Fulhorst CF, Rollin $P E$, Calaor $A B$, Manalo DL, Roces MC, et al: Epidemiology of ebola (subtype 
Reston) virus in the Philippines, 1996. J Infect Dis 1999,

179(Suppl 1):S115-S119.

28. Ikegami T, Calaor AB, Miranda ME, Niikura M, Saijo M, Kurane I, Yoshikawa Y, Morikawa S: Genome structure of ebola virus subtype Reston: differences among ebola subtypes. Brief report. Arch Virol 2001, 146(10):2021-2027.

29. Fukushi S, Mizutani T, Saijo M, Matsuyama S, Miyajima N, Taguchi F, Itamura S, Kurane I, Morikawa S: Vesicular stomatitis virus pseudotyped with severe acute respiratory syndrome coronavirus spike protein. J Gen Virol 2005, 86(Pt 8):2269-2274.

30. Saijo M, Ami Y, Suzaki Y, Nagata N, Iwata N, Hasegawa H, Ogata M, Fukushi S, Mizutani T, Sata T, et al: LC16m8, A highly attenuated vaccinia virus vaccine lacking expression of the membrane protein $B 5 R$, protects monkeys from monkeypox. J Virol 2006, 80(11):5179-5188.

\section{doi:10.1186/1746-6148-8-189}

Cite this article as: Taniguchi et al:: Analysis of the humoral immune responses among cynomolgus macaque naturally infected with Reston virus during the 1996 outbreak in the Philippines. BMC Veterinary Research 2012 8:189.

\section{Submit your next manuscript to BioMed Central and take full advantage of:}

- Convenient online submission

- Thorough peer review

- No space constraints or color figure charges

- Immediate publication on acceptance

- Inclusion in PubMed, CAS, Scopus and Google Scholar

- Research which is freely available for redistribution 